\title{
Konzeptionierung und Durchsatzanalyse eines ULD-Shuttlelagers
}

\section{Concept and Throughput Analysis of a ULD Shuttle Storage System}

\author{
Tobias Baur \\ Martin Kubaschewski \\ Frank Schönung \\ Eva-Maria Weiß \\ Institut für Fördertechnik und Logistiksysteme \\ Fakultät für Maschinenbau, Karlsruher Institut für Technologie
}

D ie Ein- und Auslagerung von Flugfrachtcontainern wird bisher üblicherweise mit Hilfe von entsprechend dimensionierten Regalbediengeräten vorgenommen. Shuttle-Systeme besitzen auch in diesem Bereich Vorteile, wie z. B. eine höhere Energieeffizienz und eine hohe Redundanz. Es wird ein Konzept zur Gestaltung eines solchen Systems vorgestellt. Ebenso wird die Leistungsfähigkeit entsprechender Lager über eine Materialflusssimulation bestimmt.

[Schlüsselwörter: ULD-Lager, Shuttlelager, Energieeffizienz, Materiaflusssimulation, Durchsatzanalyse]

$\mathbf{T}$ he storage and retrieval of airfreight containers is usually made with accordingly dimensioned storage and retrieval machines. In this industry shuttle storage systems show significant advantages as well, e.g. higher energy efficiency and a high redundancy. A concept for the construction of such a system is presented. Following the performance of corresponding warehouses is analyzed by a material flow simulation.

[Keywords: ULD-storage system, shuttle storage system, material flow simulation, energy efficiency, throughput analysis]

\section{EINLEITUNG}

In Zeiten eines stetig steigenden, weltweiten Warenumschlags und immer weiter fortschreitender Globalisierung gewinnt der Luftfracht-Sektor zunehmend an Bedeutung. Technische Fortschritte bei der Flugzeugentwicklung machten in den sechziger Jahren erstmals die kommerzielle Nutzung in großem Stil möglich, da größere und schwerere Lasten transportiert werden konnten. Seitdem verzeichnet das Flugzeug so hohe Zuwachsraten bei der Transportleistung, wie kein anderer Verkehrsträger und es wird erwartet, dass dieser Trend auch in Zukunft anhalten wird: Das Airports Council International (2007) beispielsweise prognostiziert für die transportierte Fracht bis zum Jahr 2025 eine weltweite Wachstumsrate von 5,4 \% pro
Jahr ([Sch10], S. 7). Eingesetzt wird der Luftfrachtverkehr vor allem bei Transportgütern, bei denen der Faktor Zeit eine entscheidende Rolle spielt. Um den logistischen ZeitVorteil des Flugzeugs gegenüber anderen Verkehrsträgern nicht zunichte zu machen, müssen auch die Prozesse der Flughafenlogistik in kürzester Zeit ablaufen. Dabei kommt es darauf an, den Warenumschlag im Lager durch kürzere Ein- und Auslagerzeiten zu optimieren und Standzeiten zu verringern. Schließlich haben auch die steigenden Energiekosten und Treibhausgasemissionen einen entscheidenden Einfluss auf die Flughafenlogistik: Energieeffiziente Systeme bringen ein großes Potential zur Kostenersparnis mit sich und schonen die Umwelt. Die für Unit Load Devices (ULDs), bzw. Luftfrachtpaletten bisher eingesetzten Regalbediengeräte (RBG) haben die Eigenschaft eines ungünstigen Verhältnisses von Eigenmasse zu Nutzlast und können nicht die Durchsätze moderner Shuttle-Systeme bieten.

\section{ZIELE}

Ziel des Projektes war die Entwicklung eines neuartigen Lagerkonzeptes, bestehend aus Regalanlage, Vertikalförderer, Verfahrwagen und Systemshuttle zum sicheren und effizienten Fördern und Lagern von Luftfrachtpaletten und -containern.

Die Hauptanforderungen an das System sind:

- Absolute Redundanz der Lagertechnik

- Optimale Platzausnutzung in den Regalfächern

- hoher Volumennutzungsgrad

- Varianten für mehrfachtiefe Einlagerung

- $\quad$ Ein- und Auslagerung von $100 \mathrm{ULD/h}$

- Geringere Investitionskosten

- Energieeffizienz

$\mathrm{Zu}$ fördern sind sowohl Luftfrachtcontainer als auch paletten bis zur Größe einer 10ft-Palette mit einer maximalen Nutzlast von $7.000 \mathrm{~kg}$. Der kleinste Container hat eine Größe von $1.194 \mathrm{~mm}$ x $1.534 \mathrm{~mm}$ x $1.625 \mathrm{~mm}$ 
(L x B x H), der Größte besitzt Abmessungen von $3.175 \mathrm{~mm} \times 2.235 \mathrm{~mm}$ x $1.625 \mathrm{~mm}$. Grundsätzlich muss auch die Förderung und Lagerung von Leergutcontainern möglich sein.

Da die Böden der Ladungsträger (LT) flach sind, können sie nicht unterfahren werden, wenn sie auf dem Boden stehen. Grundsätzlich muss beim Fördern stets eine sichere Fixierung der ULDs bei allen Bewegungen gewährleistet sein. Zudem sind die Böden sehr weich, weshalb ein relativ großer Teil der LT unterbaut sein muss, was sich auch in den Richtlinien der IATA (International Air Transport Association) wiederspiegelt.

\section{STAND DER TECHNIK}

\subsection{ULD-TRANSPORT}

Der Transport der ULDs erfolgt an heutigen Flughäfen meist mit zwei Techniken: Zum einen die Bereitstellung und Förderung auf Dollys. Dies sind mit Rollen ausgestattete Anhänger zum Transport der ULDs zum Flugzeug. Dort werden sie mit Hilfe eines Highloaders in das Flugzeug verladen. Zum anderen findet die Lagerung und Bereitstellung in einem automatisierten Hochregallager (HRL) statt, in dem eine doppelttiefe Lagerung möglich ist. In der Gasse zwischen jeweils zwei Regalen verfährt ein RBG, das die ULDs ein- bzw. auslagern kann und bis zu zwei 10ft-ULDs gleichzeitig aufnehmen kann. Da ein 10ft-ULD ein maximales Gewicht von $7.000 \mathrm{~kg}$ hat, muss dieses Gerät mit einer Nutzlast von 14.000 kg sehr massiv ausgeführt werden und benötigt dementsprechend eine hohe Antriebsleistung. Die Umschlagleistung eines solchen Gerätes liegt bei ca. 30 Containern pro Stunde. Die wesentlichen Nachteile dieses Systems sind unter anderem:

- $\quad$ schlechtes Verhältnis von Eigenmasse zu Nutzlast

- $\quad$ geringe Umschlagleistung.

- mangelnde Redundanz

- Hohe Kosten

Beim Be- und Entladen der LKWs auf der Landseite kommen meist mit Rollen ausgestattete Förderstrecken und an Knotenpunkten so genannte $\mathrm{x}$-y-Decks, die mit Kugeln ausgestattet sind zum Einsatz. Angetrieben werden die ULDs hier von pneumatisch anhebbaren Rollen über eine reibschlüssige Verbindung. Bei Bedarf können Sie jedoch auch von Hand bewegt werden.

\subsection{SHUTTLE-LAGER}

Shuttle-Systeme haben in den letzten Jahren zunehmend an Bedeutung gewonnen. Besonders das im Vergleich zum RBG deutlich bessere Verhältnis von Nutzlast zu insgesamt bewegter Masse bringt ein sehr hohes Energieeinsparpotential mit sich, was bei stetig steigenden Stromkosten immer wichtiger wird. Aus der möglichen
Entkopplung von Bewegungsachsen erwächst die Möglichkeit, auf mehreren Ebenen gleichzeitig Förder- und Lagerprozesse durchzuführen, wodurch eine höhere Umschlagleistung erzielt werden kann [Fra04]. Dies ist gerade im Air-Cargo-Bereich sehr wichtig, da die Bereitstellungsfristen sehr genau eingehalten werden müssen, um hohe Verzugskosten zu vermeiden.

Zwar haben bereits die meisten großen Fördertechnik-Hersteller Shuttle-Lösungen für Automatische Kleinteilelager (AKLs) und normale HRL im Produktprogramm, jedoch sind diese meist nur für eine relativ geringe Nutzlast ausgelegt. In diesem Fall muss jedoch eine Nutzlast von bis zu $7.000 \mathrm{~kg}$ bei Abmessungen von bis zu $3.175 \mathrm{~mm}$ x $2.438 \mathrm{~mm}$ transportiert werden können, was die Möglichkeiten fast aller bisher existierenden, konventionellen Konstruktionen bei Weitem übersteigt. Weltweit werden deshalb bis jetzt immer noch fast alle Luftfrachtlager mit Regalbediengeräten betrieben. Nur die Cargo Center der Lufthäfen London Heathrow und New York JFK besitzen bereits so genannte „Lift and RunSysteme“. Die Förderkomponenten solcher Systeme sind Vertikalförderer und so genannte Transfer Vehicles. Auch bei dieser Variante müssen jedoch, wie auch bei herkömmlichen RBGs, alle Lagerplätze mit Rollen ausgestattet sein.

Will man ohne Ausstattung jedes einzelnen Regalfachs mit Rollen auskommen und alle Richtlinien einhalten, so ist ein Anheben der ULDs unumgänglich. Dazu wird ein geeigneter Hubmechanismus benötigt. Für die Energiebereitstellung gibt es mehrere Möglichkeiten. Bei Systemen, bei denen die Verfahrwagen bzw. Shuttles jeweils nur auf eine Ebene beschränkt sind, ist eine Schleifleitung sinnvoll. Flexibler sind im Gegensatz dazu jedoch Systeme, die ihre Energie über Batterien oder Kondensatoren (Caps) beziehen.

\subsection{KONZEPT}

Aus mehreren erstellten Konzepten wurde nach Aspekten, die zur technischen Funktionserfüllung beitragen ein Konzept ausgewählt. Zu den Kriterien gehören:

- Durchsatz

- Effizienz

- Wirtschaftlichkeit

- Sicherheit

- Flexibilität

- Zuverlässigkeit

Das insgesamt vielversprechendste Konzept sieht vier einzelne Shuttles vor, die von einem Verfahrwagen in der Gasse bewegt werden. Sie können gemeinsam in das Fach einfahren und den Ladungsträger auf Rippen im Regal absetzen. Die Shuttles sind mechanisch unabhängig und werden während der Fahrt ins Regal von Kondensatoren mit Energie versorgt. Der Aufbau eines solchen Systems 
aus Verfahrwagen und vier Shuttles ist in Abbildung $1 \mathrm{zu}$ sehen.

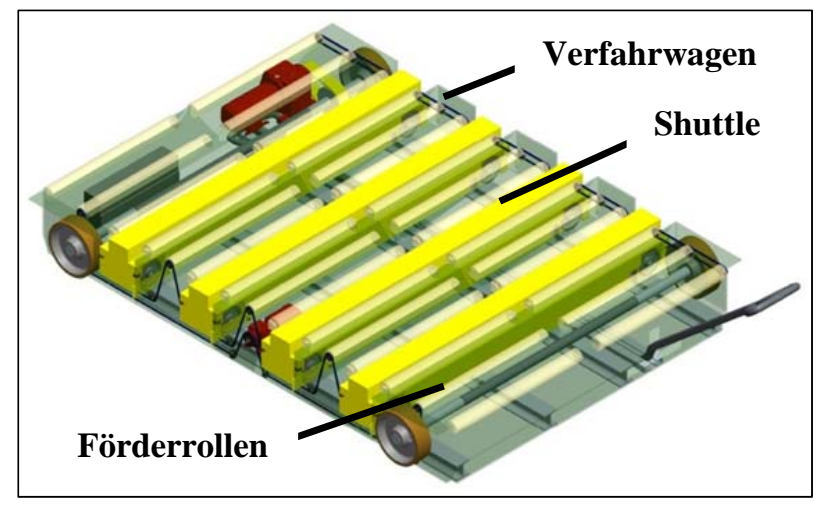

Abbildung 1. Aufbau des Verfahrwagens mit Shuttles

Da die Seiten des ULD jeweils auf dem Bodenblech aufliegen sollen, werden $n=4$ Shuttles und $n+1$ Auflageflächen des Bleches pro ULD benötigt, wobei für jedes $n$ eine Breite von $406 \mathrm{~mm}$ nicht überschritten werden darf. Um möglichst viel Raum für Antriebe und mechanische Bauteile im Shuttle zu ermöglichen, wird für die Shuttlebreite der größtmögliche Wert gewählt. Dieser liegt bei $360 \mathrm{~mm}$. So ist gewährleistet, dass es später trotzdem noch möglich ist, eventuell Lagerungen für Rollen im Fach einzubauen, die jeweils eine Breite von $20 \mathrm{~mm}$ plus $3 \mathrm{~mm}$ Luft besitzen dürften. Mit $4 * 360 \mathrm{~mm}=1.440 \mathrm{~mm}$ bleiben noch $3.175 \mathrm{~mm}-1.440 \mathrm{~mm}=1.735 \mathrm{~mm}$ für die $\mathrm{n}+1=5$ Aufstandsflächen. Für diese wird jeweils eine Breite von 372 mm gewählt um noch etwas Freiraum an den beiden Seitenflächen des ULD zu lassen. Für die Höhe des Shuttles und damit auch der Vertiefungen gibt es keine konkrete Vorgabe, jedoch wird versucht diese so gering wie möglich zu halten, um die Raumnutzung im Lager zu optimieren und die maximale Fachbodenhöhe von $500 \mathrm{~mm}$ nicht zu überschreiten. Vorerst wird die Außenhöhe auf $250 \mathrm{~mm}$ festgelegt. Die Länge des Shuttles muss mindestens die Länge eines 10ft-ULD betragen und wird auf $2.700 \mathrm{~mm}$ festgelegt.

Die Shuttles müssen in der Lage sein, den ULD mit einer geforderten Geschwindigkeit und Beschleunigung transportieren zu können. Die hierfür geforderten Werte sind in Tabelle $1 \mathrm{zu}$ sehen.

Tabelle 1. Geforderte Geschwindigkeiten der einzelnen Komponenten

\begin{tabular}{ll}
\hline Geschwindigkeit Shuttle bei Leerfahrt & $0,6 \mathrm{~m} / \mathrm{s}$ \\
\hline Geschwindigkeit Shuttle beladen & $0,3 \mathrm{~m} / \mathrm{s}$ \\
\hline Beschleunigung Shuttle bei Leerfahrt & $0,3 \mathrm{~m} / \mathrm{s}^{2}$ \\
\hline Beschleunigung Shuttle beladen & $0,3 \mathrm{~m} / \mathrm{s}^{2}$ \\
\hline Geschwindigkeit VW bei Leerfahrt & $0,8 \mathrm{~m} / \mathrm{s}$ \\
\hline Geschwindigkeit VW beladen & $0,4 \mathrm{~m} / \mathrm{s}$ \\
\hline Beschleunigung VW bei Leerfahrt & $0,5 \mathrm{~m} / \mathrm{s}^{2}$ \\
\hline Beschleunigung VW beladen & $0,5 \mathrm{~m} / \mathrm{s}^{2}$ \\
\hline
\end{tabular}

Da die Abtriebsdrehzahl der ausgewählten PolyesterUrethan-Kautschuk-Räder bei einem Raddurchmesser von $200 \mathrm{~mm}$ bei nur 28 1/min im beladenen Zustand und bei 56 1/min im unbeladenen Zustand liegt, ist ein Getriebe zur Drehzahl- und Drehmomentwandlung zwischen Motor, und Abtriebswelle notwendig. Es wird ein DrehstromAsynchron-Getriebemotor gewählt, der mit der bereitgestellten Spannung von $400 \mathrm{~V}$ betrieben wird. Um eine möglichst einfache, kostengünstige und wartungsarme Lösung zu realisieren, wird der Motor als Ausführung mit Hohlwelle direkt auf die Antriebsachse zwischen die beiden Räder gesetzt.

Ein große Herausforderung bei dem zu konstruierenden Shuttle war der Hubmechanismus, denn es ist eine Lösung notwendig, die sehr wenig Bauraum benötigt und zugleich hohe Lasten heben kann: Die maximale Nutzlast pro Shuttle ergibt sich durch Aufteilen des Maximalgewichts eines ULD auf die vier, ihn anhebenden Shuttles: $7.000 \mathrm{~kg} / 4=1.750 \mathrm{~kg}$. Um eine gewisse Toleranz gegen ungleichmäßige Beladung der ULDs zu erlauben wird ein Maximalwert von $2.000 \mathrm{~kg}$ Nutzlast pro Shuttle angenommen, also eine Gewichtskraft von rund 20.000 N, der entgegenzuwirken ist. Diese Last muss nicht nur sicher über Längere Zeit getragen werden, sondern auch in einer Zeit von 5 Sekunden abgehoben werden können. Aus den geometrischen Anforderungen im Regal ergibt sich eine aufzubringende Hubhöhe von $80 \mathrm{~mm}$, von denen $60 \mathrm{~mm}$ unter Last erfolgen. Bei einer im eingefahrenen Zustand zur Verfügung stehenden Innenhöhe von lediglich 200 mm ergibt das ein Verhältnis von Bauhöhe zu benötigtem Hubweg von 200/ 80 = 2,5. Von mehreren Detailkonzepten für den Hub hat sich eine Lösung mit Hebeln und einem Elektrozylinder der Firma SEW (Typ CMS71L) als wirtschaftlich sinnvollste Variante herauskristallisiert (siehe Abbildung 2).

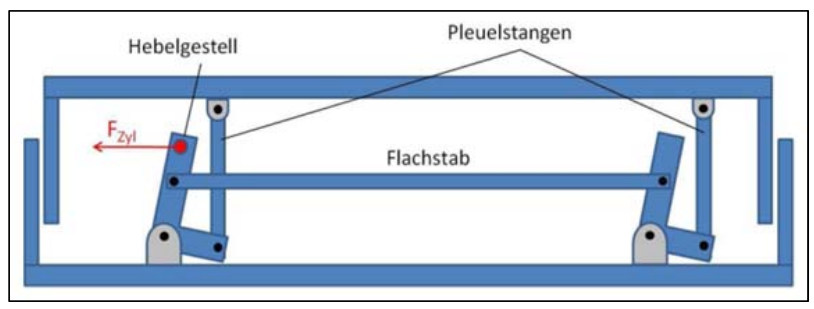

Abbildung 2. Hubvorrichtung des Shuttles

Die Energieversorgung und Steuerung der Shuttles erfolgt über eine MoviPro-Steuerung von SEW. Die Energieversorgung geschieht auf dem Verfahrwagen über Schleifleitungen. In den Regalfächern wird die benötigte Energie von Doppelschichtkondensatoren, sog. Supercaps zur Verfügung gestellt, über welche die Shuttles mit einer Spannung von $400 \mathrm{~V}$ versorgt werden.

Der Verfahrwagen weist wie das Regal Rinnen auf, in welche die Shuttles einfahren, wo Sie auch über Schleifleitungen direkt mit Energie versorgt werden. Zwischen den Shuttles sind Erhöhungen, auf denen die ULDs 
abgestellt werden können. Dort sind angetriebene Rollen verbaut, mit denen ein Verfahrwagen den Ladungsträger einem Aufzug übergeben kann. Die Aufzüge eines Systems sind so ausgeführt, dass sie zum einen die Ebenen mit ULDs ver- bzw. entsorgen können und zum anderen auch komplette Verfahrwagen auf andere Ebenen versetzen können.

Mit verschiedenen Aufzügen kann ein Shuttlesystem auf eine andere Ebene versetzt werden. Die Bewegungsachsen sind entkoppelt, d. h. Aufzüge müssen für eine Übergabe nicht auf ein Fahrzeug warten. Auf diese Weise ist das Gesamtsystem in der Lage mehrere Prozesse simultan abzuwickeln, was einen sehr hohen Durchsatz ermöglich. Zudem kann das System selbst bei einem Ausfall eines Aufzugs, eines Verfahrwagens oder von Shutteln weiterarbeiten, ist also redundant ausgeführt. Das System ist zudem noch in der Lage bei kleinen Anlagen in Kombination mit passiven Rollenbahnen die Flurfördertechnik abzubilden und damit Dollyzüge abzulösen. Dies ist allerdings noch Bestandteil weiterer Untersuchungen.

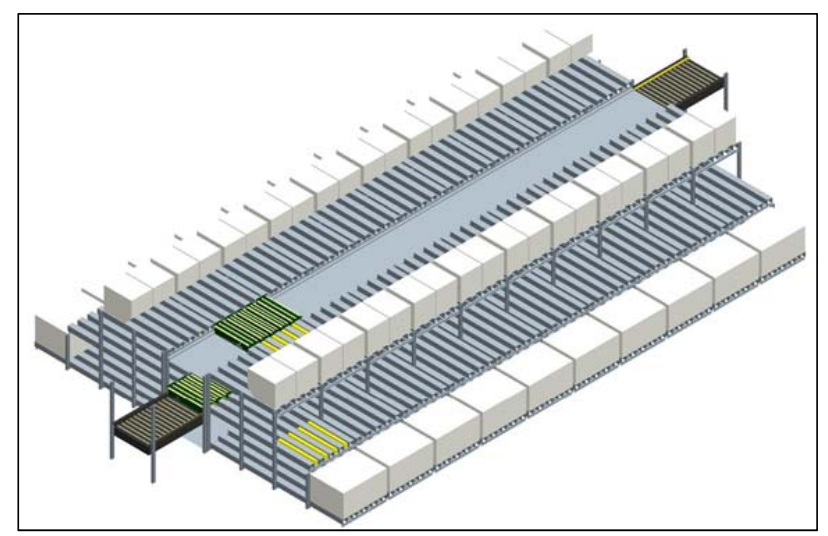

Abbildung 3: Lager mit Shuttle-System

\section{Simulation DES DURCHSATZES}

Für den Bereich hochautomatisierter Lagersysteme im Speziellen für Shuttlelager - existieren bereits eine Vielzahl an Lagersteuerungsstrategien und Modellierungsansätze in Ausrichtung auf eine simulative Systemuntersuchung. Ebenso kann bei der Strategieentwicklung für automatisierte Lager auf Formeln oder experimentelle Untersuchungen bzgl. spezifischer Lagerkennwerte, wie z. B. die Einzel- oder Doppelspielzeit, zurückgegriffen werden. Für die Entwicklung einer umfassenden Lagersteuerung sind dabei besonders die Batchbildung, die Festlegung der Reihenfolge von Ein- und Auslageraufträgen sowie die Lagerplatzvergabe von hervorgehobener Bedeutung. Routingfragestellungen sind dagegen im Normalfall nicht explizit zu behandeln, da für die Einund Auslagerung bei Vorgabe eines definierten Zielpunkts im Lager der Weg zu diesem eindeutig festgelegt ist. Bei der Platzvergabe und Reihenfolgebildung kann sich daher ausschließlich auf die Auslastung der auf diesem fest vor- gegebenen Weg befindlichen Fördermittel konzentriert werden. Des Weiteren lässt sich die Modellierung des Lagersystems auf die feste Abfolge der Fördermittel und Übergabepunkte reduzieren.

Anders verhält sich die Situation für das vorliegende neue Lagerkonzept. Es existieren dafür noch keine formal oder experimentell bestimmten Lagerkennwerte, auf deren Basis eine Steuerungsmethode entwickelt werden könnte. Zudem ist auf Grund der technischen Lagerkonzeption der Weg eines ULD durch das Lagersystem - selbst bei Vergabe eines festen Lagerplatzes - nicht eindeutig vorgegeben. Damit steht auch die Abfolge und Anzahl der in Anspruch genommenen Fördertechnik nicht von vornherein fest. Insgesamt führt dies dazu, dass der Modellaufbau für ein derartiges Lagersystem sich weitaus komplexer gestaltet und v. a. der Routingaspekt für die Entwicklung einer effizienten Steuerung an Bedeutung gewinnt.

Aus dem Fachbereich hochautomatisierter Lagersysteme bzw. im Speziellen aus dem Bereich der Shuttlesysteme können daher nur in geringem Umfang Konzepte der Modell- und Steuerungsgestaltung für das vorliegende ULD-Shuttlelager adaptiert werden. Vielmehr muss auf eine allgemeine Betrachtung des Lagers als Materialflusssystem zurückgegangen werden, um eine geeignete Modellierungs- und Steuerungsvariante identifizieren und evaluieren zu können. Der Ansatz für eine derartige Darstellung liegt dabei in der Modellierung des Lagersystems als gerichteter Graph bzw. Netzwerk. Es wird versucht, alle erforderlichen Systemeigenschaften des ULD-Lagers in dem Netzwerk zu repräsentieren und dabei auch auf übertragbare Ansätze aus verwandten Fachbereichen, wie manuellen Materialflusssystemen oder Transportnetzwerken, zurückzugreifen.

\subsection{MODELLIERUNG DES NETZWERKES}

Auf die Netzwerkmodellierung wird auch im Rahmen der Steuerungsentwicklung zurückgegriffen, v. a. bei der Auswahl eines geeigneten, auf die Durchsatzmaximierung des Gesamtsystems ausgelegten Routingverfahrens. Zugleich wird mit der Strategiekonzeption bzgl. des Batchings, der Reihenfolgebildung und der Lagerplatzvergabe auf einen Ausgleich der schwankenden Auftragseingänge und auf eine möglichst gleichmäßige Auslastung des Lagerequipments abgezielt. Wie bereits dargelegt, kann für das vorliegende Lagersystem dabei nur bedingt auf existierende Literaturansätze zurückgegriffen werden. Daher besteht der Ansatz darin, die Strategien zunächst ausschließlich basierend auf der Analyse der Lagerprozesse zu entwickeln und anschließend im Rahmen der Simulation zu evaluieren sowie ggf. anzupassen.

In dem Lager-Simulationsmodell wurden der Modellierungsansatz sowie das Steuerungskonzept umgesetzt und Auswertungsfunktionen bezüglich der lagerspezifischen Kennwerte integriert. Anschließend wurden mit dem Modell verschiedene Layoutvarianten des ULD- 
Lagers bzgl. definierter Durchsatzniveaus simuliert und damit erste Rückschlüsse auf die Leistungsfähigkeit des Systems und die Vorteilhaftigkeit verschiedener Layoutvarianten gezogen. Des Weiteren dienten die Ergebnisse dazu, die entwickelte Steuerungslogik bzgl. ihrer Zielsetzung zu evaluieren und optimale Layout und Strategiekombination zu identifizieren.

Eine Anforderung bzgl. des vorliegenden Lagersystems war es, das System in seinen Dimensionen variabel zu halten. Um dementsprechend eine adaptive Kapazitätszuweisung und Kantenbewertung zu erzeugen, wurde für das vorliegende Lagersystem in Anlehnung an Busacker ([Bus04], S.72 ff.) eine Struktur entwickelt, bei der sich das Netzwerk aus Knoten und Kanten zusammensetzt, die jeweils die Kapazität von genau eines ULD aufweisen. Über diese, auch als „Rasterung“ bezeichnete Einteilung des Netzwerks in einheitlich dimensionierte Abschnitte, lassen sich auch die Transferzeiten zwischen zwei Rastereinheiten standardisiert berechnen. Auf Grund der regelmäßigen Lagerstruktur kann das Netzwerk aus diesen standardisierten Rastereinheiten für verschiedene Layouts automatisch generiert werden.

In hochautomatisierten Lagersystemen, wie in RBGHochregallagern oder in Kanal-Shuttle- Systemen, ist für gewöhnlich der Transportweg einer Lagereinheit zwischen E/A-Bereich und einem vorgegebenen Lagerplatz eindeutig vorbestimmt. Ebenso ist bekannt, welches Lagerequipment während des Transports in Anspruch genommen wird. Routingverfahren spielen daher für die Steuerung derartiger Lagersysteme keine Rolle. Anders sieht es bei dem vorliegenden ULD-Shuttlesystem aus. Durch die Möglichkeit, die ULDs sowohl über die Rollenfördererbahnen der untersten Ebene als auch über freie Shuttlekanäle sowie mehrere Lifte hinweg zu befördern, ergibt sich eine Vielzahl an unterschiedlichen Transportrouten zwischen den E/A-Bereichen und den Lagerplätzen. Für ein effizientes und exaktes Routing müssen neben der zeitabhängigen Auslastung auch die Transferbedingungen zwischen den einzelnen Fördermitteln darstellbar sein. Zu diesen Bedingungen zählt zum Beispiel die Einschränkung, dass das ULD von einem Rollenförderer nur dann auf den Verfahrwagen abgegeben werden kann, wenn dieser an dem entsprechenden Übergabepunkt bereitsteht. Die dabei ggf. auftretenden Wartezeiten bis zum Eintreffen des Verfahrwagens müssen daher ebenso aus dem Netzwerk ermittelbar sein wie die eigentlichen Transportzeiten. Das Routing über drei Knoten mit der Einplanung von Transferzeiten und dem Belegen von entsprechenden Zeitfenstern ist in Abbildung 4 dargestellt.

\subsection{LAGERPLATZVERGABE UND ROUTING}

Für die drei Aspekte - Batching, Scheduling und Lagerplatzvergabe - bestehen in Bezug auf die vorliegende ULD-Lagertechnik bisher noch keine Ansätze in der Fachliteratur (vgl. [Rou00] und [Gu07]). Auch das Über- tragen komplexerer Strategien, die speziell für andere, hoch-automatisierter Lagersysteme, wie z. B. für RBGHochreggallager oder AKL-Shuttlesysteme eingesetzt werden, war nicht möglich. Dies liegt daran, dass die für die Adaption der Strategien notwendigen Formeln oder experimentellen Bestimmungen von Systemparametern, wie z. B. Spiel oder Bedienzeiten, für das neu-konzipierte Lagersystem noch komplett fehlen. Des Weiteren lagen zum Zeitpunkt der Modellerstellung noch keine zuverlässigen Informationen darüber vor, ob und in welchem Umfang routingrelevante Daten eines ULD bereits bei Systemeintritt bekannt sind. $\mathrm{Zu}$ diesen Daten zählen z. B. der erwartete Auslagerzeitpunkt, Informationen über das Ziel der Auslagerung sowie über weitere Einheiten, die zusammen mit dem ULD ausgelagert werden sollen. Das Vorhandensein oder Fehlen dieser Informationen bestimmt jedoch im Wesentlichen das Spektrum anwendbarer Strategien. Dementsprechend erschien es als wenig zielführend, ausgereifte Strategien für diese Steuerungsbereiche zu entwerfen, die rein auf Annahmen bzgl. der tatsächlichen Informationslage basieren.

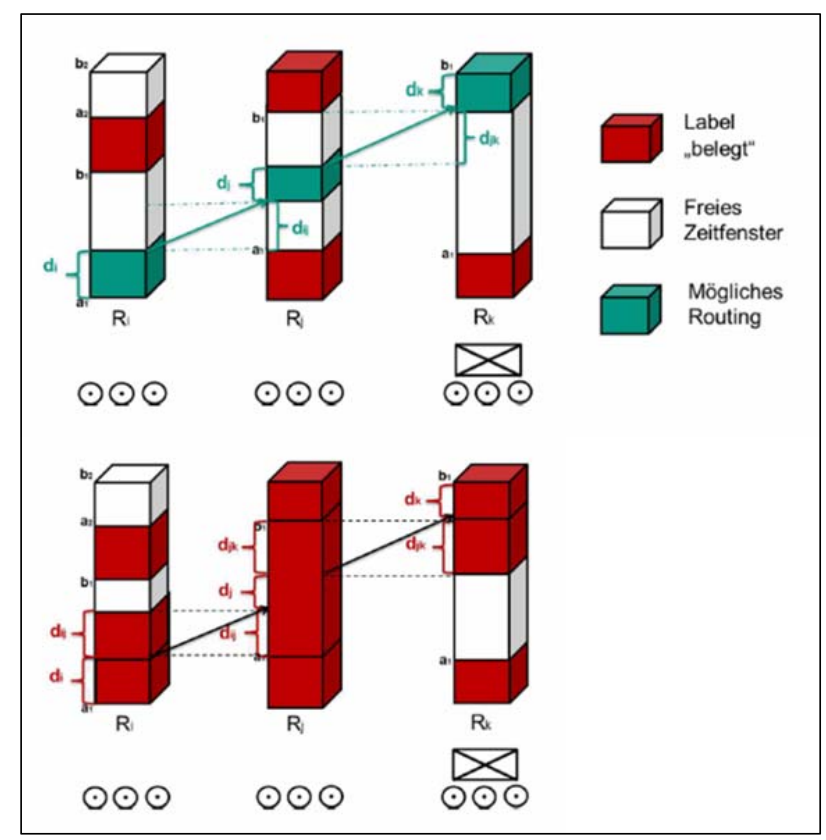

Abbildung 4. Zeitfenster beim Routing über mehrere Knoten

Deshalb wurden zunächst einfache Steuerungsstrategien basierend auf der Analyse vorteilhafter Lagerprozesse sowie logischen Überlegungen konzipiert. Die Strategieentwicklung für das Batching und Scheduling sollte sich hierbei primär auf die Anpassung der Steuerung an die stark schwankende Auftragslage des Luftfrachtbereichs fokussieren. Bei der Lagerplatzvergabe stand v. a. das Erzielen kurzer Ein- und Auslagerzeiten sowie die Vermeidung von durchsatzsenkenden Umlageroperationen im Vordergrund.

Die drei Steuerungsbereiche, das Batching, die Reihenfolgebildung sowie die Lagerplatzvergabe, werden auf 
Grund ihrer abgestimmten Konzeption in ihrem gemeinsamen Wirken beschrieben. Zum einen wird davon ausgegangen, dass für die ankommenden Einlageraufträge sowohl die Informationen über den geplanten Auslagerzeitpunkt also auch über den E/A-Bereich (Land- bzw. Luftseite), über den sie das Lager verlassen, bereits vorhanden sind. Zum anderen wird bezüglich der Vorsortierung und Batcheinteilung von Einlageraufträgen angenommen, dass ankommende ULDs, die das Lager über den gleichen E/A-Bereich verlassen und deren Auslagerzeitpunkt ungefähr im gleichen Zeitraum liegt, sich zu Batches von jeweils drei ULDs zusammenfassen lassen.

Die Lagerplatzvergabe erfolgt jeweils immer für einen gesamten Dreier-Batch. Dabei unterscheidet sich die Strategie in der untersten Ebene von der, die in den oberen Ebenen angewandt wird. Bei ersterer wird versucht, die ULDs möglichst in Kanälen mit geringem Abstand zu der Achse einzulagern, die die Lagerfläche in eine linke und rechte Hälfte unterteilt. Durch diese Platzvergabe sollen die Wege, die der Verfahrwagen zwischen zwei Einlageraufträgen in der Gasse zurücklegen muss, möglichst kurz gehalten werden. Auch wird in einem Kanal immer zuerst der innere, an die Verfahrwagengasse direkt angrenzende, Platz vergeben. Würde der äußere Platz zuerst vergeben, so könnte der innere Lagerplatz nur über die Verfahrwagengasse erreicht werden. Dies führt insgesamt zu einer längeren Belegung des Verfahrwagens und des Shuttles für den Einlagerauftrag, was im Rahmen der Durchsatzmaximierung zu vermeiden ist. Dabei ist auch darauf zu achten, dass die Reihenfolgebildung innerhalb des Batches so erfolgt, dass zuerst das auf dem äußeren Lagerplatz befindliche ULD wieder ausgelagert wird. Auf Grund der doppelttiefen Lagerung in der untersten Lagerebene kann es dazu kommen, dass in einem Kanal ULDs für zwei verschiedene Dreier-Batches eingelagert werden. Jedoch wird auch in diesem Fall versucht, die äußere Einheit zuerst auszulagern, falls für diese bereits ein Einlagerauftrag vorliegt.

Ebenso wurde im Rahmen der Steuerungskonzeption eine „Einbahnstraßenregelung“ für die Rollenförderer der untersten Ebene eingeführt, um durch die gleichgerichtete Bewegungsführung der ULDs eine durchsatzsteigernde Wirkung zu erzielen. Die Richtung der Einbahnstraße wurde dahingehend ausgelegt, dass v. a. die aus den oberen Ebenen über die Lifte ausgeschleusten Einheiten danach eine möglichst kurze Strecke über die Rollenförderer bis zum Lagerausgang zurückzulegen haben.

\subsection{SimulationSAUFBAU}

Die Simulation des ULD-Shuttlelagers wurde mittels der Simulationssoftware AnyLogicTM der Firma XJ Technologies implementiert. Generell unterstützt die Software drei Simulationsparadigma - System Dynamics (SD), Discrete Event Simulation (DES) und Agent Based Modeling (ABM). Für die Simulation des ULD-Lagers wurde die Modellierung gemäß dem DES-Paradigmas vorgenommen. Die Programmierung der Lagersteuerung sowie die Klassen- und Methodendefinitionen in AnyLogic erfolgt komplett in der Programmiersprache Java.

Die Simulationsszenarien wurden dahingehend entworfen, dass sie möglichst gut verschiedene Gesamtlagerdimensionen und Lagerlayouts repräsentieren. Die dabei entstandenen vier Basisszenarien sind in Tabelle 2 ersichtlich.

\section{Tabelle 2. Festlegung der Simulationsszenarien}

\begin{tabular}{lllll}
\hline Szenario & $\mathbf{1}$ & $\mathbf{2}$ & $\mathbf{3}$ & $\mathbf{4}$ \\
\hline Ebenanzahl & 2 & 3 & 3 & 4 \\
\hline Anzahl Lagerkanäle & 15 & 10 & 19 & 14 \\
Anzahl Lagerplätze & 150 & 160 & 304 & 308 \\
Maximaler Durchsatz [1/h] & 56,82 & 67,71 & 54,46 & 59,00 \\
\hline Lagerfüllgrad & $70 \%$ & $70 \%$ & $70 \%$ & $70 \%$ \\
\hline
\end{tabular}

Für alle Szenarien wurde basierend auf deterministisch gestalteten Auftragsdaten der Maximaldurchsatz bei 70\% Lagerfüllgrad durch die Simulation ermittelt. Die deterministischen Auftragsdaten wurden dabei so konzipiert, dass die Anzahl eingehender Ein- und Auslageraufträge von Airside zu Landside ungefähr 2:1 beträgt. Basis für die Annahme bzgl. des Auftragsverhältnisses waren Projektdaten des Industriepartners FAB GmbH. Bezüglich dieser vier Basisszenarien und der ermittelten Maximaldurchsätze wurden für jedes Szenario drei Unterszenarien erzeugt. Diese sollten dazu dienen, das Systemverhalten bei stochastischer Auftragsankunft zu simulieren, wenn im Durchschnitt dabei die Auftragsdaten zu einem Durchsatz von $50 \%$, $70 \%$ sowie $90 \%$ des Maximaldurchsatzes führen.

\subsection{ERGEBNISSE DER SiMULATION}

Anhand von Szenario 1 und Szenario 3 wurde nun das Verhalten der Ein- und Auslagerdauer bzgl. der unterschiedlichen Durchsatzhöhen verglichen. Abbildung 5 verdeutlicht grafisch die Auswertungsergebnisse der beiden Basisszenarien.

Eindeutig zu erkennen ist für beide Szenarien, dass sowohl die Netto- als auch die Bruttoeinlagerdauer mit steigendem Durchsatz stetig zunehmen, wobei eine Steigerung von $50 \%$ auf $70 \%$ des Maximaldurchsatzes eine geringere Auswirkung auf beide Zeiten hat als der Anstieg von $70 \%$ auf $90 \%$. Allgemein lässt sich der Zeitanstieg durch die mit dem steigenden Durchsatz einhergehende höhere Auslastung der Fördermittel und dem durchschnittlichen Einlagerweg einer ULD erklären. Gemäß der Einbahnstraßenregelung bzgl. der Rollenförderer, müssen alle ULDs über einen Teil oder - für alle Einheiten, die in die oberen Lagerebenen gehen - über den gesamten Rollenfördererabschnitt zwischen dem Lagereingang und dem Umsetzer vor dem entsprechend nächstgelegenem Lift, befördert werden. 


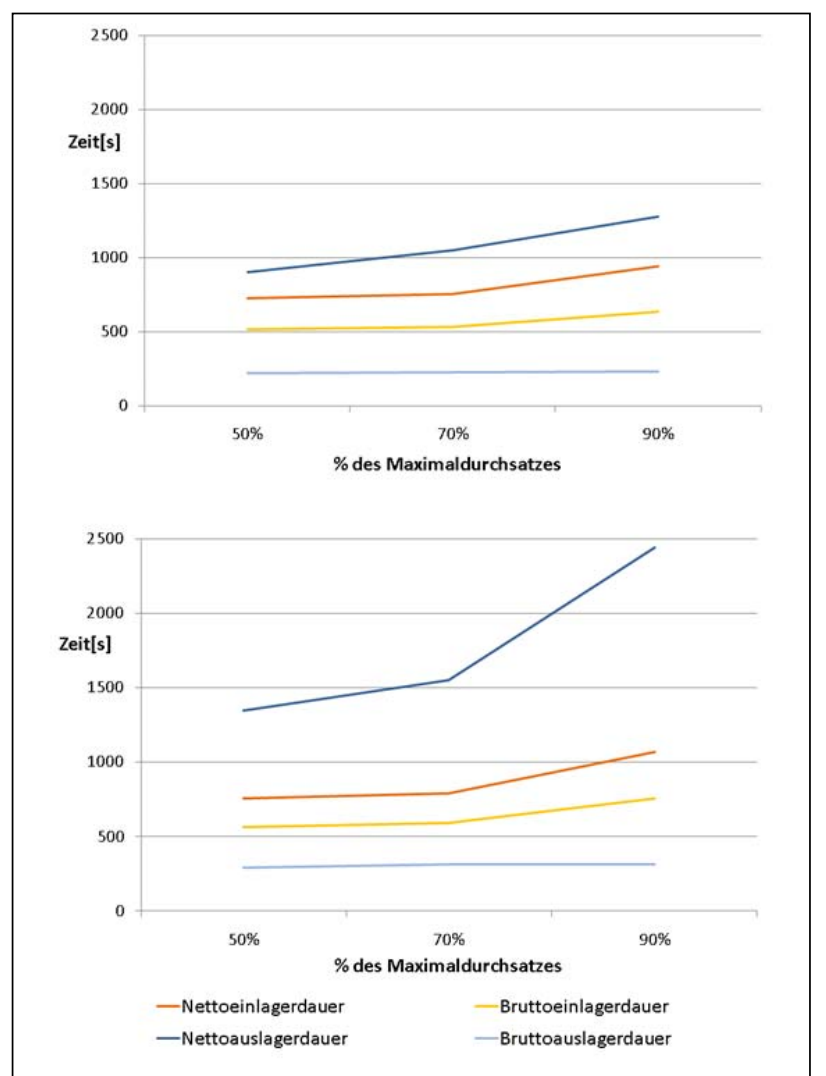

Abbildung 5. Analyse der Ein- und Auslagerdauern für Szenario 1 und Szenario 3

Abbildung 6 zeigt den deutlich messbaren Auslastungsunterschied auf den betroffenen Rollenfördererabschnitten zwischen der Situation bei 50\% und 90\% der Maximalauslastung. Auch die an den Rollenförderertransfer anschließenden Transportprozesse über Lift sowie Verfahrwagen und Shuttle sind auslastungsabhängig, da eine Einheit erst von diesem Fördermittel aufgenommen werden kann, wenn alle vorherigen Aufträge abgearbeitet wurden. Die Anzahl an zuvor abzuarbeitenden Aufträgen steigt dementsprechend mit der Auslastung. Da diese Wartezeit bis zur Aufnahme durch den Verfahrwagen oder Lift in beide Lagerzeiten - die Netto- und die Bruttoauslagerzeit - mit eingeht, erklärt sich deren proportionaler Kurvenverlauf.

Zunächst zeigt sich die eingeführte Einbahnstraßenregelung bezogen auf die Bruttoauslagerdauer als besonders vorteilhaft, da sie zu Auslagerzeiten führt, die nur marginal von der Systemauslastung abhängen. Diese Eigenschaft ist besonders wichtig, da die Auslagerung auf Luftseite den zeitkritischsten Prozess darstellt. Bisher wurde für das Lagersystem keine Prioritätsunterscheidung zwischen Ein- und Auslageraufträgen getroffen, woraus sich die lange Nettoauslagerdauer ergibt. Hier wäre ein erster Ansatz, eine Priorisierungsstrategie zu entwerfen, die - ohne den Gesamtdurchsatz negativ zu beeinflussen Auslageraufträgen eine höhere Dringlichkeit zuweist und somit die Nettoauslagerdauer senkt. Dabei muss eine län- gere Einlagerdauer der Aufträge in Kauf genommen werden. Ebenso zeigt die Auslastungsuntersuchung der Fördermittel, dass noch nicht über alle Szenarien hinweg die Auslastung der Verfahrwagen- und Shuttleeinheiten in den verschiedenen Ebenen ein ausgeglichenes Niveau erreicht. Ein Einbezug aktueller Systemauslastungen in die Lagerplatzvergabe wäre hier anzudenken.

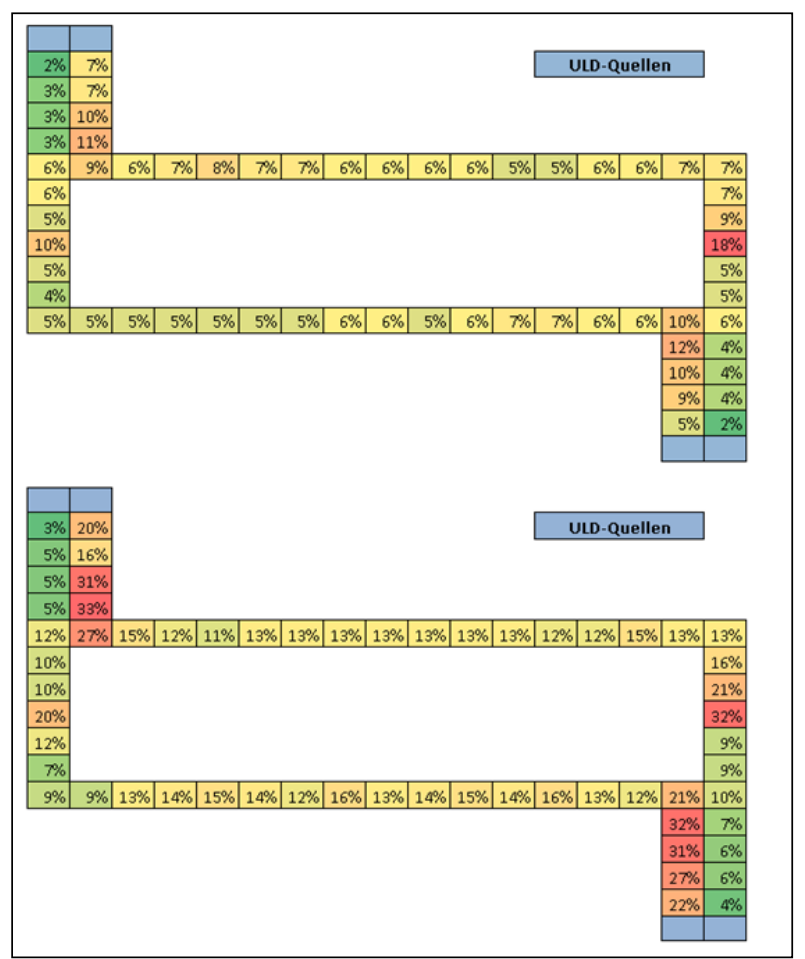

Abbildung 6. Auslastung der Rollenförderer für Szenario 1, bei $50 \%$ und $90 \%$ des Gesamtdurchsatzes

\section{LITERATUR}

[Fra04] Fraunhofer: Shuttle machen Lager flexibel, Fraunhofer Magazin, Band 2, 2004.

[Gu07] Gu, Jinxiang; Goetschalckx, Marc; McGinnis, Leon F.: Research on warehouse operation: A comprehensive review, European Journal of Operational Research 177 (1), 2007, S. 1-21

[Rou00] Rouwenhorst, B.; Reuter, B.; Stockrahm, V., van Houtum, G. J.; Mantel, R. J.; Zijm, W. H. M.: Warehouse design and control: Framework and literature review, European Journal of Operational Research 122 (3), 2000, S. 515-533

[Sch10] Schlegel, Aandreas: Bodenabfertigungsprozesse im Luftverkehr. Gabler Verlag, 2010

[Bus04] Busacker, Torsten: Steigerung der FlughafenKapazität durch Modellierung und Optimierung von Flughafen-Boden-Rollverkehr. Dissertation, Technische Universität Berlin, 2004 